\title{
Protocol for Light-Dark and Activity Rhythm Therapy for sleep: Feasibility and acceptability in Schizophrenia spectrum disorders (L-DART FitSz)
}

Sophie Faulkner ( $\sim$ 1sophiefaulkner@gmail.com )

Greater Manchester Mental Health NHS Foundation Trust https://orcid.org/0000-0003-1549-0922

\section{Penny E. Bee}

UoM: The University of Manchester

Richard J. Drake

UoM: The University of Manchester

\section{Study Protocol}

Keywords: Circadian rhythm, insomnia, psychosis, mental health, occupational therapy, actigraphy, wearables, light therapy, dawn simulation, behaviour change

Posted Date: April 19th, 2021

DOI: https://doi.org/10.21203/rs.3.rs-426609/v1

License: @ (1) This work is licensed under a Creative Commons Attribution 4.0 International License. Read Full License 


\section{Abstract}

Background

Sleep problems are common in people with diagnoses of schizophrenia spectrum disorders $(>50 \%)$, even during periods of relative stability of psychotic symptoms. Evidence suggests that people living with schizophrenia spectrum disorders are often keen to improve their sleep, but few non-pharmacological sleep treatments are available to patients in specialist mental health services. It has been proposed that occupational therapists may have the relevant skills for the delivery of behavioural sleep interventions. This mixed method, proof-of-concept study aims to assess the feasibility and acceptability of a new intervention, Light-Dark and Activity Rhythm Therapy (L-DART), to improve sleep in people with schizophrenia spectrum disorder diagnoses.

Methods

A single group of 10 service users with schizophrenia spectrum diagnoses and self-reported problems with sleep onset, maintenance, timing or quality will be offered L-DART. L-DART will be delivered over 6-9 in person sessions and 3-6 phonecalls by an occupational therapist. Feasibility measures will comprise recruitment and retention logs, fidelity based on session records, adverse effects, and study attrition. Intervention uptake, engagement and adherence will be measured, and barriers to adherence explored. Acceptability will be assessed though quantitative satisfaction ratings and qualitative interviews. Activity patterns and dynamic light exposure will be measured, as well as self-reported sleep, wellbeing and functioning, to inform outcome selection in a larger trial.

Discussion

The findings will inform any necessary modifications to the intervention and its materials, enabling the development of a stage 2 manual and a therapist training package. The results will support the design of a randomised multi-therapist feasibility trial.

Trial registration

ISRCTN11998005, assigned registration on 17.02.2020

\section{Background}

Sleep problems are common in people with schizophrenia spectrum disorders (schizophrenia, schizoaffective disorder and delusional disorder) (1), and cause, or are linked to, significant problems with physical and mental health (2-5). Sleep problems have been linked to poorer medication adherence (6), and circadian rhythm (body clock) problems are thought to be responsible for some of the cognitive impairment associated with schizophrenia (7).

People with psychotic illnesses see sleep as important and want to sleep better (8); participants with schizophrenia have reported that poor sleep impacts on their functioning and mental health and feel that poor sleep should be better treated (9). Participants describe the impact of sleep problems on their self-image, on their ability to keep social arrangements and their ability to obtain paid or voluntary work (10). Experts identify quality of sleep as a modifiable factor and an important treatment target in schizophrenia $(7,11)$, yet sleep treatment is rarely offered to those under specialist mental health services. Staff often lack knowledge and confidence to intervene $(12,13)$.

People with schizophrenia have somewhat different sleep problems than those in the general population and those with common mental health problems, in whom psychophysiological insomnia is common. People with schizophrenia experience more circadian dysregulation (less regular day-night rhythms) (14), often resulting in reduced daytime activity, and increased night-time activity, irregular or changeable rest-activity rhythms, or non $24 \mathrm{hr}$ (free-running) patterns $(1,15) .50 \%$ have been found to have significantly delayed, or free running rest-activity patterns. People with schizophrenia described that broken sleep, or sleep which was not experienced as 'deep', was a very high priority to improve (10). Broken or shallow sleep is also a very common experience by both subjective accounts and objective measurement $(10,16-18)$.

Qualitative research suggests patients prefer non-drug to drug interventions for sleep difficulties, despite the effort they acknowledge is required to successfully engage in these $(9,10,19)$. Patient and public involvement completed whilst designing this programme of research has corroborated this stance.

Page 2/18 
Current theories of sleep regulation suggest reducing excessive time in bed, reducing napping, and increasing daytime activity (thereby increasing 'sleep pressure' and tiredness at bedtime) are all strategies likely to improve sleep maintenance (20). Cognitive Behavioural Therapy for Insomnia (CBT-i) includes elements to manipulate sleep pressure, to increase behavioural associations of bed with sleep, as well as sleep hygiene advice $(21,22)$. Many protocols also include strategies to reduce arousal or address night-time worry, such as relaxation or cognitive therapy elements (22).

Increasing daytime activity can improve timing of exposure to environmental and behavioural cues (zeitgebers) including light, which help to entrain circadian rhythms and keep bodily sleep-wake rhythms aligned with day-night cycles $(11,23)$. This study will evaluate an intervention which includes more increased focus on these environmental zeitgebers, compared to traditional CBT-i, delivered by an occupational therapist.

Authors have described the relevance of the occupational therapist's existing skill set, to sleep interventions; including: activity analysis, activity scheduling, graded behaviour change interventions, and environmental assessment and adaptation (13,24-26). Sleep is increasingly becoming recognised as a legitimate concern for occupational therapists and receiving increased coverage in literature (27), and therapist training (28). However a scoping search of completed or ongoing occupational therapy trials in schizophrenia found none related to sleep. This study is the first we are aware of to develop an occupational therapy intervention to address sleep in this group.

\section{Light-Dark and Activity Rhythm Therapy for sleep: Development of the L-DART intervention}

A Delphi study was conducted by the study authors (October 2018 - March 2019) to systematically identify and integrate the views and expertise of clinical and academic experts and people with lived experience (service users and carers). The findings of this study were used to develop the intervention which will be tested in this study.

L-DART incorporates some elements of cognitive behavioural therapy for insomnia: sleep scheduling, avoiding or reducing naps and increasing association of bed with sleep through 'stimulus control'. L-DART also utilises current knowledge of circadian rhythm, changing light exposure patterns to phase advance or phase delay, and stabilising circadian rhythm and rest-activity patterns. L-DART frames and personalises light and activity level and timing modifications in the context of meaningful and balanced activity routines (meaningful occupation), utilising the core skills of occupational therapy.

The intervention addresses the following core areas:

- modifications to light exposure patterns across the day including environmental adaptation

- nature, balance and timing of activities

- sleep schedule modifications

Previous research, and Delphi study, suggests a high level of personalisation is required to treat sleep in this client group, more so due to the diversity of sleep disturbance phenotypes. These include delayed/advanced sleep, non24hr rhythms, irregular sleep timing, psychophysiological insomnia, insomnia due to poor sleep hygiene, and 'hypersomnia' with poor sleep quality. There are thus different approaches depending on the problem, and optional components to address specific sleep disrupting influences which may be present for some but not all participants.

\section{Aims of the present study:}

This study aims to recruit 10 participants, who will all receive the intervention. Our aim is to assess and explore acceptability and feasibility, this will be approached using mixed methods. We will also measure potential clinical outcomes around sleep and quality of life, which would be measured in a larger future study.

\section{Methods}

This protocol paper corresponds to approved research protocol v1.3, 15.01.2021

\section{Primary aim:}

To explore the feasibility and acceptability of delivering and testing L-DART within a larger scale trial. 
To evaluate or provide:

- To what extent participants find the intervention acceptable

- Qualitative exploration of acceptability

- Whether participants are willing and able to adhere to different aspects of the intervention.

- Initial exploration of the proposed mechanisms of action, and problem types and combinations for which different elements of the intervention are more or less applicable.

- Mixed methods evaluation of adherence and barriers to adherence.

- Feasibility of recruitment and resources required to recruit.

- A rough estimate of rates of attrition likely in a larger study (although confidence intervals for this estimate are likely to be wide (Hertzog 2008)).

- Initial indications of effect size

- Suitability of outcome measures and passive data monitoring (activity and light measurement devices):

- Their ability to capture factors participants deemed important (via comparison with qualitative data).

- Acceptability of measures, participant burden, face validity and perceived relevance.

- Levels of completion on measures.

- Initial indications of variance on selected measures (although this would require supplementation with further crosssectional work (Thabane et al. 2010).

- Relationships and comparability of data from self-report measures and passive data channels to inform which are most useful in a larger future study.

- To contribute toward estimates of the number of potentially eligible participants.

\section{Sample:}

Target sample $=10$

Sample size is not based around efficacy or estimation of effects, but seeks to balance feasibility of study delivery within the available timescale and resources, whilst recruiting a sufficient number of participants to represent a range of different types of sleep problems and circumstances. A purposive approach (29) will be used if some problem types are becoming under-represented by mid-

study, by requesting certain types of referrals from gatekeepers, but participants will not be denied participation in order to purposively sample.

Inclusion criteria:

1. Diagnosis of non-affective psychosis: Schizophrenia, Schizotypal disorder, Delusional disorder, Schizoaffective disorder (ICD-10 F20, F21, F22, F25, F28 / DSM 295.*, 297.1).

2. Open to secondary care mental health services in Greater Manchester Mental Health and NHS Foundation Trust (GMMH) or Pennine Care NHS Foundation Trust (Pennine Care).

3. Over 18 .

4. Expresses dissatisfaction with their sleep (length of time to fall asleep, amount of sleep, subjective sleep quality, broken sleep, unrefreshing sleep, difficulty waking up, unsatisfactory timing of sleep).

5. Interested in receiving the intervention.

\section{Exclusion criteria:}

Stability or acuity:

1a. Change of medication within the last 1 month. 
1b. Discharged from hospital within the last 1 month.

1c. Current inpatient or acuity of illness requiring home treatment team.

1d. Actively suicidal (expressing suicidal plans or intent).

1e. Risk to others prevents lone visiting.

Due to wrong sleep disorder:

2a. Known untreated significant sleep apnoea (Apnoea Hypopnoea Index (AHI) Index $>20$, or symptomatic)

2b. Primary complaint is of sleep apnoea, sleep related movement disorder or parasomnia.

2c. Diagnosis of narcolepsy or Rapid Eye Movement (REM) sleep behaviour disorder.

1. Co-morbid learning disability, dementia, or moderate to severe neurological impairment.

2. Alcohol or substance dependent (unsuitable if using heavily every day and/or not able to be sober for sessions).

3. No fixed abode.

4. Does not have capacity to give informed consent.

\section{Recruitment:}

Service user participants will be recruited via clinical gatekeepers who will be approached at team meetings, one to one in teams, and via other Trust research promotion (e.g. internal email, 'splash-screen' adverts). Leaflets can be passed on by gatekeepers. Service user participants will be approached by members of the research delivery team at GMMH or Pennine where local policies permit this. The researcher will attend client and service user meetings by arrangement or invitation to present information about the study. Posters and leaflets will be placed in patient areas so that potential participants can self-refer. The referral form will be used by research delivery staff, clinical staff or the chief investigator to record / pass on the details of potential participants who have verbally consented to be contacted by the chief investigator.

\section{Participants who withdraw consent [or lose capacity to consent]:}

Participants can withdraw consent at any time without giving any reason, as participation in the research is voluntary, without their care or legal rights being affected.

If a participant loses the capacity to consent during the study (for instance due to acute physical or mental illness), we will retain the data already contributed, but not collect any new data. When (or if) he participant regains capacity to consent we will ask if they want to continue to participate further.

\section{Data collection:}

Routine therapy data:

- Initial assessment proforma

- Self-reported activities (diary)

- Self-reported sleep (diary)

- Homework log (plan, progress \& feedback)

- Therapy notes, correspondence \& documents (emails, letters, therapy summary and maintenance plan)

- Adverse effects monitoring (checklist)

Custom measures and case report forms:

- Therapy satisfaction 3 item Likert 
- Qualitative pre-intervention questions regarding - prospective acceptability ( $\sim 5$ minutes)

- Qualitative post-intervention interview - retrospective acceptability ( 60minutes)

- End point assessment questions

- Demographic information form

- Therapy component delivery and fidelity log

- Attendance log and adherence log

- Recruitment log

\section{$\underline{\text { Standardised outcome measures: }}$}

- Insomnia Severity Index (ISI) $(30,31)$

- PROMIS-SD 8a (Sleep Disturbance) $(32,33)$

- PROMIS-SRI 8a (Sleep Related Impairment) $(32,33)$

- Warwick-Edinburgh Mental Wellbeing Scale (WEMWBS) (34)

- EQ 5D-5L (5-level EQ-5D version, EuroQol) (35)

- PROMIS-AP 8a (Ability to Participate in social roles and activities) (36)

- Clinical Global Impression-Schizophrenia (CGI-SCH) (37)

Passive data / objective data:

- Withings Move activity watch data -

- Walking and running: Steps \& pace (we will not collect any Global Positioning System (GPS) data, distance and elevation using GPS will only be collected by HealthMate if the person has chosen to turn on connected GPS, which we are not asking people to do)

- Swimming: Session duration

- Automatic detection of: Walking, Running, Cycling, Tennis, Table Tennis, Squash, Badminton, Weights, Basketball, Soccer, Volleyball, Dance, Boxing (time \& duration)

- Sleep: Deep and light sleep phases, sleep interruptions

- CamnTech MotionWatch 8 accelerometry and light exposure recording (wrist worn, Dynamic outdoor light - coat/bag mounted, environmental light - mounted in the home)

- Therapy session audio recordings (additional consent, not essential to participation)

\section{Therapy satisfaction 3 item Likert:}

Custom measure to be used during and after intervention, give 5 point ratings on 3 questions relating to affective attitude toward the intervention - liking, satisfaction, readiness to recommend.

\section{Pre-intervention questions - prospective acceptability:}

There will be a brief ( 5minutes) qualitative exploration of prospective perceived effectiveness, and self-efficacy completed by the chief investigator pre-intervention (see qualitative topic guide).

\section{Qualitative post-intervention interview:}

The post-intervention interview will be conducted by an experienced qualitative researcher with lived experience of psychosis and of receipt of mental health services. The interview will explore various dimensions of acceptability of the therapy as a whole and of its various components (see qualitative topic guide).

\section{End point assessment questions:}


Repetition of the quantitatively rated and self-reported objective questions from the initial assessment proforma, used to quantify change.

\section{Demographic information form:}

Record of demographic information used to describe the sample and to inform the therapy. Information collected after consent to participation but prior to the start of therapy from clinical notes or from referrer. Items included to describe sample: diagnosis, gender, age at day 1 of study, ethnicity, religious / spiritual orientation. Items also used within initial assessment: height, weight, BMI (to inform screening for sleep disordered breathing), medications, doses, timing and frequency.

\section{Therapy component delivery and fidelity log:}

Record of which components have been delivered and when to each participant, and any changes or protocol deviations which occurred (and why), completed by the therapist after the session.

\section{Attendance and adherence}

Records of sessions attended within the therapy window, and of homework completion between sessions by participants, completed by the therapist at and after sessions.

\section{Recruitment log}

Record of referrals with consent to contact received, sources of referrals, and reasons for ineligibility or reasons for declining participation (where reasons were given).

See Figure 1 for the research participation timeline per participant, and Table 1 for an overview of which measures are used at each time point.

\section{Results}

Table 1: Data collection per week of participation: 


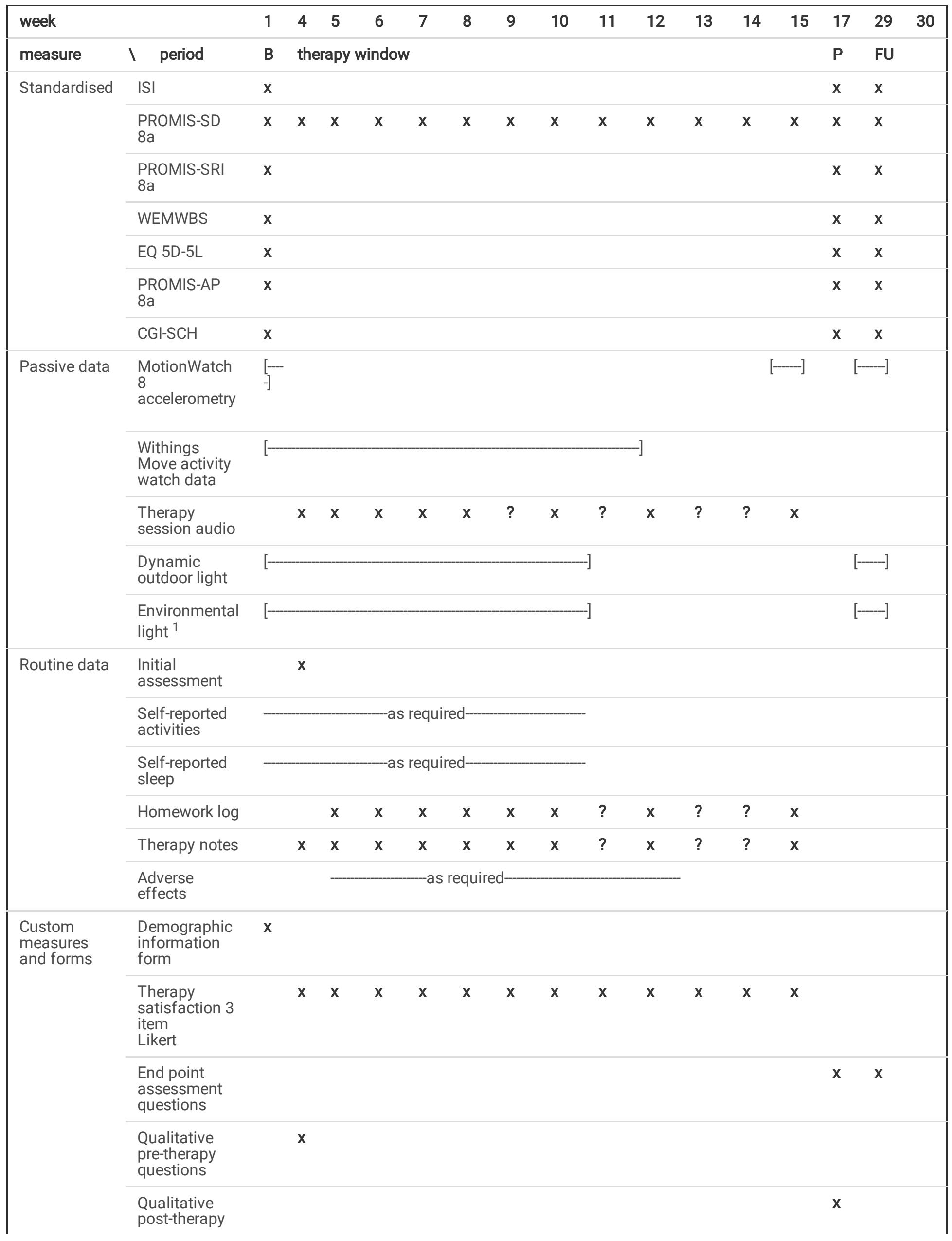


interview

$\begin{array}{lllllllllllll}\begin{array}{l}\text { Therapy } \\ \text { delivery and } \\ \text { fidelity log }\end{array} & \mathbf{x} & \mathbf{x} & \mathbf{x} & \mathbf{x} & \mathbf{x} & \mathbf{x} & \mathbf{x} & \mathbf{x} & \mathbf{x} & \mathbf{x} & \mathbf{x} & \mathbf{x} \\ \begin{array}{l}\text { Attendance } \\ \text { and } \\ \begin{array}{l}\text { adherence } \\ \text { records }\end{array}\end{array} & \mathbf{x} & \mathbf{x} & \mathbf{x} & \mathbf{x} & \mathbf{x} & \mathbf{x} & \mathbf{x} & \mathbf{x} & \mathbf{x} & \mathbf{x} & \mathbf{x} & \mathbf{x} \\ \begin{array}{l}\text { Recruitment } \\ \text { log }\end{array} & \mathrm{n} / \mathrm{a} \text {, data collected prior to recruitment }\end{array}$

$\mathrm{B}=$ baseline, $\mathrm{P}=$ post-intervention, $\mathrm{FU}=$ follow up, ?=dependent on occurrence of session/ phone-call (optional), ${ }^{1}$ At week 1 or at first home visit, prior to modification to home environment \& following modification to home environment or at end of intervention.

\section{Intervention:}

The study is a single group design, all participants will be offered L-DART.

L-DART will be delivered by the Chief Investigator $(\mathrm{Cl})$ who is an experienced mental health occupational therapist, it will be given in 69 sessions plus 3-6 phone calls as needed.

The intervention addresses the following core areas:

- modifications to light exposure patterns across the day

- nature, balance and timing of activities

- sleep schedule modifications

Optional components to be used when relevant include:

- reducing or changing the timing of use of substance use including: caffeine, alcohol, illicit drugs, over the counter medications

- altering or regulating the timing of sleep-inducing prescribed medications

- addressing meal timing

- addressing nightmares

- methods to reduce or avoid daytime naps

See Figure 2 for an overview of core and optional components and when used.

1) Modifications to light exposure

Modern light exposure patterns differ from those in which humans evolved, due to time spent indoors and exposure to artificial light at night. These light patterns are more difficult for the circadian system to entrain to, and, due to individual differences, some are affected more than others (38). L-DART will aim to return light exposure patterns closer to those which naturally occur on earth (i.e. light in the daytime and dark at night), by increasing daytime and morning light exposure, and reducing artificial light at night and in the late evening.

For those with a late-shifted rhythm there will be more emphasis on increasing morning light and avoiding evening light, to bring the rhythm earlier. For those with an early-shifted rhythm (less common) there will be more emphasis on daytime and afternoon light exposure, and less emphasis on reducing evening light. We will not increase evening light to attempt to delay sleep patterns, as metaanalysis has not shown evidence for this (39), and as light exposure is acutely alerting evening light exposure may interfere with sleep $(40,41)$. For those with an irregular sleep rhythm, true circadian timing (e.g. timing of melatonin rhythm) cannot easily be ascertained without more invasive procedures than we plan to use, so daytime light and late-evening and night-time light avoidance will be emphasised, as this should be beneficial irrespective of circadian timing at baseline. 
What is useful to address will vary depending on the baseline light exposure patterns of each individual, for instance some participants will already have plenty of daytime light and so might only need to reduce evening light. Typical changes will include:

Behavioural changes, such as:

- Going outdoors more.

- Sitting in a different place indoors where there is more natural light.

- Travelling by a different means to get more day light, e.g. walking rather than driving

- Reducing use of light emitting screens in the evening and at night.

- Switching from main lights to dimmer lamps in the evening.

Environmental modification and provision of equipment:

- Provision of blackout curtains in the bedroom to reduce night-time light from street lights.

- Provision of lace/net curtains to allow curtains to be opened in the daytime without compromising privacy (windows overlooked by neighbours and passers-by).

- Moving furniture to enable sitting / working in areas with more natural light where possible.

- Fitting dim bulbs in lamps in order to allow switching to lower light in the evening.

- Provision of a sunrise alarm.

- Provision of a light box with instructions for use when natural light cannot be obtained (e.g. poor weather, limited mobility, symptoms of illness which make it difficult to go out). Use will be in accordance with manufacturers guidelines and will supplement natural and environmental light exposure where required.

2) Nature, balance and timing of activities

Depending on participants occupational routine at baseline we will address occupational imbalance, occupational deprivation, timing of occupations, and light exposure achieved during occupations. This is assessed in the initial assessment, and through wearable activity and light data. Depending on the situation this might involve changing the timing of existing activities, changing locations activities are done, introducing new activities, and potentially reducing engagement in some activities. An interests checklist will be used where participants would benefit from input to identify activities they might enjoy. Participants' values, preferences, interests and goals will be taken into account, as well as their desired changes to their sleep-wake pattern. Collaborative graded goals will be set, activity scheduling will be used, and progress monitored through self-report and output from activity and light sensors. Participants will be encouraged to monitor their own progress with physical activity and activity timing goals through the L-DART app, which will show them their activity from the Withings Move.

\section{3) Sleep schedule modifications}

The sleep opportunity window (time in bed) will be modified if needed to improve its regularity, length, and timing in relation to social and personal requirements. For some this will involve shortening the length of time in bed if it is overly long, for some this will involve increasing regularity of timing, for some this will involve making a gradual shift in timing - if sleep timing is regular, but too early or too late. For those whose timing and length of time in bed is already appropriate, but who are unable to get to sleep and sleep through, a temporary and gradually titrated shortening of the sleep window will be used (sleep compression), in order to increase sleep pressure and improve sleep onset and maintenance, and associations of bed with sleep. The ' 15 minutes rule' (get out of bed if you have been awake for 15 minutes) or variants of this (using a longer period than 15 minutes, get out of bed 'if you are frustrated', or 'if you don't feel at all sleepy') will be discussed, and plans made will depend on the participant's preferences and presentation. The instruction to 'go to bed only when feeling sleepy' will be added only after evening wind down routine has been addressed, if still needed.

\section{Research and therapy devices}

Movement sensing, light sensing, and light emitting devices will be used within the research study and the intervention. All devices have been tested by the manufacturers prior to being made available on the market, all are available for individual consumers to purchase on the open market without prescription.

Page $10 / 18$ 
Although the light boxes are used outside of their CE marked purpose, and this study is not designed or powered to support any additional CE marking of these light boxes. None of the devices are the subject of the study, but are used as tools within the study to monitor activity and light, and to modify light exposure. See Table 2.

Devices will be issued directly to participants by the research therapist.

Table 2: Devices used within the study

\begin{tabular}{|c|c|c|c|}
\hline Device type & $\begin{array}{l}\text { Device brand } \\
\& \text { model }\end{array}$ & Classification and certified use of device & Use within this study \\
\hline $\begin{array}{l}\text { Light box } \\
\text { (medium size) }\end{array}$ & $\begin{array}{l}\text { Lumie } \\
\text { Arabica }\end{array}$ & \multirow{2}{*}{$\begin{array}{l}\text { Class 11a Medical Device, CE marked for to } \\
\text { improve energy circadian rhythm and mood in } \\
\text { winter depression (seasonal affective } \\
\text { disorder). }\end{array}$} & \multirow{2}{*}{$\begin{array}{l}\text { Use as per manufacturer instructions, but } \\
\text { outside of CE mark in schizophrenia } \\
\text { spectrum disorder. Two size options for } \\
\text { practicality. }\end{array}$} \\
\hline $\begin{array}{l}\text { Light box } \\
\text { (small size) }\end{array}$ & $\begin{array}{l}\text { Lumie } \\
\text { Vitamin L }\end{array}$ & & \\
\hline Wake up light & $\begin{array}{l}\text { Phillips } \\
\text { Somneo }\end{array}$ & $\begin{array}{l}\text { Class } 1 \text { Medical device, CE marked for use to } \\
\text { provide a more gradual wake up and better } \\
\text { morning alertness. }\end{array}$ & $\begin{array}{l}\text { Use as per manufacturer instructions and } \mathrm{CE} \\
\text { mark. }\end{array}$ \\
\hline $\begin{array}{l}\text { Activity } \\
\text { tracking } \\
\text { watch } \\
\text { (consumer } \\
\text { wearable) }\end{array}$ & $\begin{array}{l}\text { Withings } \\
\text { Move }\end{array}$ & $\begin{array}{l}\text { Not classified as a medical device, classified } \\
\text { as a wellbeing wearable. }\end{array}$ & $\begin{array}{l}\text { For participants to self-monitor their activity } \\
\text { patterns when making changes as part of } \\
\text { the intervention. }\end{array}$ \\
\hline $\begin{array}{l}\text { Actigraphy } \\
\text { watch } \\
\text { (research } \\
\text { device) }\end{array}$ & $\begin{array}{l}\text { CamnTech } \\
\text { Motionwatch } \\
8\end{array}$ & $\begin{array}{l}\text { Class } 1 \text { Medical device, CE marked for use to } \\
\text { measure activity, sleep and light within } \\
\text { research. }\end{array}$ & $\begin{array}{l}\text { Used as a research outcome measure. } \\
\text { Light exposure measurements used as part } \\
\text { of initial assessment. }\end{array}$ \\
\hline
\end{tabular}

\section{Analysis:}

\section{Feasibility of therapist delivery of the intervention, and participant adherence to the intervention:}

Therapy component delivery and fidelity logs will be summarised regarding therapist's protocol non-adherence using descriptive statistics. Reasons recorded for non-adherence, and components in which non-adherence occurred will be examined. Although there is some flexibility in order of delivery and optional components, therapist fidelity issues could include: failure to deliver the core content, delivery of components in some way differently to the protocol, delivery content outside of its allowed time range, or significant time spent not delivering intervention content at all (for instance this can occur if managing immediate risk, where no intervention content might be delivered that session).

Attendance at sessions will be summarised using descriptive statistics, as will reasons for missed sessions (e.g. client cancelled/postponed in advance, client did not attend, session not arranged (client unavailable), session not arranged (therapist unavailable).) Completion of the minimum number of sessions will be summarised.

Rates of completion of delivery of the core components of the intervention will be summarised, and time and sessions taken to deliver these. Contact time to deliver the intervention in total will be summarised.

Participant adherence to different elements of the homework will be summarised, including:

- watching/reading educational content.

- making self-report recordings.

- making behavioural changes / completing agreed goals (sub-categories relating to area of change, e.g. sleep timing, caffeine, screen use, daytime activities).

Qualitative reports regarding barriers and facilitators of adherence will be explored within the qualitative interviews. 
Qualitative data will be compared within and between participants regarding aspects or components of the intervention which were better or worse adhered to, and these will be examined in relation to acceptability (for instance, did some participants not adhere to a certain component because it conflicted with their values, or because they did not see it as important?).

We will calculate what modifications in activity levels, light exposure patterns, and other 'intermediate' outcomes have been taken place during the therapy, by comparing data from the activity tracking devices, light sensors, and self-report measures before and after intervention. Where appropriate comparisons to baseline values will use statistical tests for paired data.

We will make preliminary examinations of whether in individual cases and across cases, the mechanisms of change may be as proposed by background theory and expert opinion - as represented in the logic model. For instance if the end outcome occurs, but the intermediate outcomes we supposed were responsible for causing that outcome never occurred for any participants, then this will prompt discussion, potentially revision of the logic model, and could prompt design of further exploratory or mechanism based research.

\section{Acceptability of the intervention}

Likert ratings of satisfaction will be summarised using descriptive statistics and graphed for each participant and will accompany the qualitative acceptability data and week by week therapy component delivery log. We will examine whether there are any particular therapy components whose delivery are associated with higher or lower ratings of satisfaction by examining satisfaction average ratings on weeks during and after these are given, and by triangulating with qualitative data.

Semi-structured qualitative interviews will be analysed in Nvivo software, coding inductively using thematic analysis $(42,43)$, and also coding to an a priori descriptive framework (e.g. regarding topics discussed) (44). Transcripts will be separately analysed by the qualitative interviewer researcher and by the $\mathrm{Cl}$. An a-priori coding frame will be developed containing descriptive codes, and further analytic codes will be developed during analysis.

\section{Feasibility of larger scale testing of the intervention, and future implementation:}

Rates of eligible participants will be summarised, as will number of declines, with reasons given (or no reason given). Recruitment rates per month and by the end of the study will be summarised. Attrition from the study will be summarised both during the intervention and from follow up.

Problem sub-types, problem clusters or phenotypes for which the intervention is more or less adhered to or more or less effective this will be examined by reference to qualitative data predominantly as quantitative data will be underpowered to detect differences between groups, however quantitative data will also be examined alongside the qualitative data.

Pre-post change and variance will be calculated for each measure in order to begin to inform future sample size calculations. Outcome measures (both content and change captured) will be compared to the quantitative data regarding factors participants deemed important, in order to inform selection of measures and selection of a primary outcome for a future larger study. Acceptability of measures, face validity and perceived relevance, will be evaluate will be ascertained through analysis of the qualitative interview. Participant burden of measures will be ascertained from the qualitative interview and also through timing completion. Levels of completion on measures will be summarised, and any particular items or measures with higher rates of noncompletion will be noted (with reasons if these can be ascertained from researcher notes or the qualitative interviews).

Self-report and passive data channels will be examined in order to inform which are most practical and useful in a larger future study, and for use within the intervention.

Time taken to deliver components, and to deliver the intervention overall, will be examined in case efficiencies or problems can be identified. Recruitment logs will be examined regarding reasons for ineligibility and therapy related reasons for declines (when reason given), as these could affect future implementation in clinical practice as well as larger scale testing (45).

\section{Safety considerations and adverse events}

It is not anticipated that any aspect of L-DART poses a high risk of harming participants, however the potential risks include: 
- Eye strain / discomfort

- Headache

- Migraine (in those who experience migraine)

- Sunburn (going outdoors)

- Risk of falls (reducing evening illumination)

- Distress when discussing sleep or something related to sleep

- High or low energy and mood

Eye-stain, or a headache should go away if the person stops using the light box. Some people get used to the light box so that it no longer causes them discomfort, or sit farther away to reduce the brightness.

Light therapy boxes don't cause sunburn, but increasing time outdoors could. We will talk to participants about how to avoid sunburn when we talk about going outside more.

Reducing evening illumination and installation of blackout curtains poses the potential to increase risk of falls, this will be reduced by advising participants on how to ensure sufficient light to safely move around at night (for instance using the 'midnight light' mode on the Phillips Somneo wake-up light for night-time bathroom trips), and by encouraging clear thoroughfares without trip hazards.

With an increase in light exposure comes a potential risk of hypomania or agitation, however light therapy using light boxes has been used in studies in bipolar without this causing mania. Participants with schizoaffective disorder will be gradually and cautiously introduced to light exposure. Participants mental state and functioning will be monitored when time in bed is being shortened. Participants will be advised not to drive or operate heavy machinery if they feel sleepy.

\section{Adverse effects monitoring and recording:}

Participants will be asked about any adverse effects they might have experienced from any aspect of the intervention at each session by the therapist, and these will be recorded on the adverse effects checklist, including notes regarding to what the effect is attributed (e.g. from use of the light box, from sleep schedule changes).

\section{Adverse Events monitoring and reporting}

Adverse effects and adverse events will be monitored and recorded, these will be discussed routinely at intervals with the therapist researcher's clinical academic supervisor Richard Drake. More serious adverse events (whether intervention related or not) will be reported and discussed with Richard Drake as soon as practicably possible. Decisions and categorisations will be made regarding severity and potential intervention relatedness, and where ambiguity or uncertainty exists, cases will be discussed with an independent expert (Professor Bill Deakin). Serious Adverse Events (SAEs) will be reported to the Research Ethics Committee if they are intervention related and unexpected.

\section{Discussion And Conclusions}

The study was paused due to covid-19 and is now due to commence recruitment in early 2021. Delivery of the intervention entirely online was considered, however this was not done due to aspects which would not translate readily to remote delivery such as provision of equipment, home assessment, and practical aspects such as addressing window coverings. Acceptability and feasibility of a remote delivery variant is an interesting but distinct question.

Sleep problems in people with diagnosis of schizophrenia spectrum disorder require treatment as part of routine care. If a relatively brief and cost-effective sleep treatment can be evidenced, recommended and implemented, this has the potential to improve quality of life and functional outcomes for this group. Cost savings elsewhere in services due to reduced relapse are also a real possibility. The findings of this study will form the first stage in this process, by informing any necessary modifications to the intervention and its materials, enabling the development of a stage 2 manual, and a therapist training package. If feasible, the results will support design of a larger scale multi-therapist feasibility study. Findings of this study will be disseminated via a peer reviewed journal, and to study participants and referrers in a more accessible format. 


\section{Abbreviations}

L-DART - Light-Dark and Activity Rhythm Therapy

OT - occupational therapist

GMMH - Greater Manchester Mental Health and NHS Foundation Trust

AHI - Apnoea Hypopnoea Index

REM - Rapid Eye Movement

ISI - Insomnia Severity Index (ISI)

PROMIS - Patient Reported Outcomes Measurement Information System

WEMWBS -Warwick-Edinburgh Mental Wellbeing Scale

CGI-SCH - Clinical Global Impression-Schizophrenia

SAE - Serious Adverse Events

GPS - Global Positioning System

NHS - National Health Service

ICD-10 - International Classification of Diseases 10

\section{Declarations}

\section{Ethics approval and consent to participate}

HRA and Health and Care Research Wales (HCRW) Approval was received on 13.03.2020, following a favourable opinion by North West - Greater Manchester South Research Ethics Committee, REC reference: 20/NW/0059. The study sponsor is University of Manchester (fbmhethics@manchester.ac.uk).

\section{Consent for publication}

Not applicable

\section{Availability of data and materials}

Data sharing is not applicable to this article as no datasets were generated or analysed during the current study.

\section{Competing interests}

The authors declare that they have no competing interests

\section{Funding}

This report is independent research arising from a Clinical Doctoral Research Fellowship, awarded to Sophie Faulkner (NIHR award identifier: ICA-CDRF-2016-02-007) supported by the National Institute for Health Research and Health Education England. The views expressed in this publication are those of the authors and not necessarily those of the NHS, the National Institute for Health Research, Health Education England or the Department of Health.

\section{Authors' contributions}

The idea was conceived by SF, and prepared for submission for funding with supervisory input of PB \& RD. The study was designed by SF with advice from PB \& RD. The protocol, and this protocol manuscript, were each written by SF and reviewed edited and 
commented on by PB \& RD, then revised by SF.

\section{Acknowledgements}

Acknowledgements goes to Margaret Ogden, and Anonymous, who were involved in commenting on aspects of the study design and intervention design throughout, as well as to other individual patient and public contributors who gave one off input. Acknowledgement goes to the Service User Reference Group (SURG) at Psychosis Research Unit (PRU), GMMH NHS FT, who commented on study materials including the Participant Information Leaflet, and regarding intervention naming. Acknowledgement goes to Derk-Jan Dijk, who was involved at the stages of funding application, intervention design, and study design.

Acknowledgement goes to Bill Deakin for agreeing to act as an independent expert for reporting of adverse events. Acknowledgement goes to University of Manchester for the loan of Actigraphy and light monitor devices are. Acknowledgement goes to Lumie who provided light boxes for study participants. Acknowledgement goes to Adrian Harwood who developed the L-DART app.

\section{Authors' information}

SF is an experienced mental health occupational therapist, who began to research sleep in 2013 after noting the severe impact on occupational functioning of the irregular and reversed sleep cycles of some service users, and the lack of non-pharmacological interventions available.

\section{References}

1. Wulff K, Dijk D-J, Middleton B, Foster RG, Joyce EM. Sleep and circadian rhythm disruption in schizophrenia. Br J Psychiatry [Internet]. 2012 Apr [cited 2014 Oct 20];200(4):308-16. Available from: http://www.pubmedcentral.nih.gov/articlerender.fcgi? artid $=3317037 \&$ tool $=$ pmcentrez\&rendertype $=$ abstract

2. Krystal AD, Thakur M, Roth T. Sleep Disturbance in Psychiatric Disoders: Effects on Function and Quality of Life in Mood Disorders, Alcoholism, and Schizophrenia. Ann Clin Psychiatry. 2008;20(1):39-46.

3. Spiegel K, Knutson K, Leproult R, Tasali E, Van Cauter E. Sleep loss: a novel risk factor for insulin resistance and Type 2 diabetes. J Appl Physiol [Internet]. 2005 Nov [cited 2014 Nov 4];99(5):2008-19. Available from:

http://www.ncbi.nlm.nih.gov/pubmed/16227462

4. Meerlo P, Sgoifo A, Suchecki D. Restricted and disrupted sleep: effects on autonomic function, neuroendocrine stress systems and stress responsivity. Sleep Med Rev [Internet]. 2008 Jun [cited 2014 Sep 16];12(3):197-210. Available from:

http://www.ncbi.nlm.nih.gov/pubmed/18222099

5. Haus EL, Smolensky MH. Shift work and cancer risk: potential mechanistic roles of circadian disruption, light at night, and sleep deprivation. Sleep Med Rev [Internet]. 2013 Aug [cited 2014 Oct 25];17(4):273-84. Available from:

http://www.ncbi.nlm.nih.gov/pubmed/23137527

6. Afonso P, Brissos S, Cañas F, Bobes J, Bernardo-Fernandez I. Treatment adherence and quality of sleep in schizophrenia outpatients. Int J Psychiatry Clin Pract [Internet]. 2014 Jan [cited 2014 Oct 20];18(1):70-6. Available from:

http://www.ncbi.nlm.nih.gov/pubmed/24047426

7. Wulff K, Joyce E. Circadian rhythms and cognition in schizophrenia. Br J Psychiatry. 2011;198(4):250-2.

8. Auslander LA, Jeste D V. Perceptions of problems and needs for service among middle-aged and elderly outpatients with schizophrenia and related psychotic disorders. Community Ment Health J [Internet]. 2002;38(5):391-402. Available from: http://ovidsp.ovid.com/ovidweb.cgi?T=JS\&PAGE=reference\&D=emed5\&NEWS=N\&AN=2004163305

9. Waite F, Evans N, Myers E, Startup H, Lister R, Harvey AG, et al. The patient experience of sleep problems and their treatment in the context of current delusions and hallucinations. Psychol Psychother Theory, Res Pract [Internet]. 2015;89:181-193. Available from: http://doi.wiley.com/10.1111/papt.12073

10. Faulkner S, Bee P. Experiences, perspectives and priorities of people with schizophrenia spectrum disorders regarding sleep disturbance and its treatment: a qualitative study. BMC Psychiatry [Internet]. 2017;17(1):158. Available from: DOI 10.1186/s12888-017-1329-8

11. Jagannath A, Peirson SN, Foster RG. Sleep and circadian rhythm disruption in neuropsychiatric illness. Curr Opin Neurobiol [Internet]. 2013 [cited 2014 Nov 4];23(5):888-94. Available from: http://dx.doi.org/10.1016/j.conb.2013.03.008 
12. O'Sullivan M, Rahim M, Hall C. The prevalence and management of poor sleep quality in a secondary care mental health population. J Clin Sleep Med. 2015;11(2):111-6.

13. Faulkner S, Mairs $\mathrm{H}$. An exploration of the role of the occupational therapist in relation to sleep problems in mental health settings. Br J Occup Ther [Internet]. 2015;78(8):516-24. Available from: http://bjo.sagepub.com/lookup/doi/10.1177/0308022614564771

14. Bersani FS, lannitelli A, Pacitti F, Bersani G. Sleep and biorythm disturbances in schizophrenia, mood and anxiety disorders: A review. Riv Psichiatr. 2012;47(5):365-75.

15. Eklund M, Erlandsson L, Leufstadius $\mathrm{C}$. Time use in relation to valued and satisfying occupations among people with persistent mental illness: Exploring occupational balance. J Occup Sci. 2010;17(4):231-8.

16. Ilanković A, Damjanović A, Ilanković V, Filipović B, Janković S, llanković N. Polysomnographic sleep patterns in depressive, schizophrenic and healthy subjects. Psychiatr Danub. 2014;26(1):20-6.

17. Afonso P, Figueira ML, Paiva T. Sleep-wake patterns in schizophrenia patients compared to healthy controls. world J Biol psychiatry [Internet]. 2014;15:517-24. Available from: http://www.ncbi.nlm.nih.gov/pubmed/23316764

18. Faulkner S, Drake R, Dijk D-J, Bee P. Development of an occupational therapy intervention to improve sleep in people with schizophrenia spectrum disorders. IRAS ID 228454. Unpubl Rep. 2019;

19. Faulkner S, Bee P. Perspectives on Sleep, Sleep Problems, and Their Treatment, in People with Serious Mental IIInesses: A Systematic Review. PLoS One [Internet]. 2016;11(9):e0163486. Available from: doi.org/10.1371/journal.pone.0163486

20. Kyle SD, Aquino MRJ, Miller CB, Henry AL, Crawford MR, Espie CA, et al. Towards standardisation and improved understanding of sleep restriction therapy for insomnia disorder: A systematic examination of CBT-I trial content. Sleep Med Rev. 2015;23:83-8.

21. Miller CB, Espie CA, Epstein DR, Friedman L, Morin CM, Pigeon WR, et al. The evidence base of sleep restriction therapy for treating insomnia disorder. Sleep Med Rev. 2014;18(5):415-24.

22. Matthews EE, Arnedt JT, McCarthy MS, Cuddihy LJ, Aloia MS. Adherence to cognitive behavioral therapy for insomnia: A systematic review. Sleep Med Rev [Internet]. 2013;17(6):453-64. Available from: http://dx.doi.org/10.1016/j.smrv.2013.01.001

23. Pauley SM. Lighting for the human circadian clock: recent research indicates that lighting has become a public health issue. Med Hypotheses [Internet]. 2004 Jan [cited 2014 Nov 4];63(4):588-96. Available from:

http://www.ncbi.nlm.nih.gov/pubmed/15325001

24. Fung C, Wiseman-Hakes C, Stergiou-Kita M, Nguyen M, Colantonio A. Time to wake up: bridging the gap between theory and practice for sleep in occupational therapy. Br J Occup Ther [Internet]. 2013;76(8):384-6. Available from: http://openurl.ingenta.com/content/xref?genre=article\&issn=0308-0226\&volume=76\&issue=8\&spage=384

25. Solet JM. Sleep and rest. In: Schell BA, Gillen G, Scaffa M, Cohn ES, editors. Willard and Spackman's Occupational Therapy. 12th ed. Philladephia: Wolters Kluwer - Lippincott Williams and Wilkins; 2014. p. 714-30.

26. Creek J. Occupational therapy defined as a complex intervention. London: College of Occupational Therapists; 2003.

27. Green A, Brown C. An Occupational Therapist's Guide to Sleep and Sleep Problems. London: Jessica Kingsley Publishers; 2015.

28. Brown C. Pre-Conference Workshop: The Occupation of Sleep [Internet]. 2016 [cited 2016 May 2]. Available from: http://www.caot.ca/default.asp?pageid=3984

29. Teddlie C, Yu F. Mixed Methods Sampling: A Typology With Examples. J Mix Methods Res. 2007;1(1):77-100.

30. Bastien C, Valliers A, Morin CM. Validation of the Insomnia Severity Index as an outcome measure for insomnia research. Sleep Med. 2001;2:297-307.

31. Morin CM, Belleville G, Bélanger L, Ivers H. The insomnia severity index: Psychometric indicators to detect insomnia cases and evaluate treatment response. Sleep. 2011;34(5):601-8.

32. Yu L, Buysse DJ, Germain A, Moul DE, Stover A, Dodds NE, et al. Development of Short Forms From the PROMIS ${ }^{T M}$ Sleep Disturbance and Sleep-Related Impairment Item Banks. Behav Sleep Med. 2011;10(1):6-24.

33. Buysse DJ, Yu L, Moul DE, Germain A, Stover A, Dodds NE, et al. Development and validation of patient-reported outcome measures for sleep disturbance and sleep-related impairments. Sleep [Internet]. 2010;33(6):781-92. Available from: https://www.ncbi.nlm.nih.gov/pmc/articles/PMC2880437/

34. Tennant R, Hiller L, Fishwick R, Platt S, Joseph S, Weich S, et al. The Warwick-Edinburgh mental well-being scale (WEMWBS): Development and UK validation. Health Qual Life Outcomes. 2007;5:1-13.

Page 16/18 
35. Janssen MF, Pickard AS, Golicki D, Gudex C, Niewada M, Scalone L, et al. Measurement properties of the EQ-5D-5L compared to the EQ-5D-3L across eight patient groups: A multi-country study. Qual Life Res. 2013;22(7):1717-27.

36. Hahn EA, DeVellis RF, Bode RK, Garcia SF, Castel LD, Eisen S V., et al. Measuring social health in the patient-reported outcomes measurement information system (PROMIS): Item bank development and testing. Qual Life Res. 2010;19(7):1035-44.

37. Haro JM, Kamath SA, Ochoa S, Novick D, Rele K, Fargas A, et al. The Clinical Global Impression-Schizophrenia scale: A simple instrument to measure the diversity of symptoms present in schizophrenia. Acta Psychiatr Scand Suppl. 2003;107(416):16-23.

38. Skeldon AC, Phillips AJK, Dijk D-J. The effects of self-selected light-dark cycles and social constraints on human sleep and circadian timing: a modeling approach. Sci Rep [Internet]. 2017;7(45158). Available from:

http://www.nature.com/articles/srep45158

39. Faulkner SM, Bee PE, Meyer N, Dijk DJ, Drake RJ. Light therapies to improve sleep in intrinsic circadian rhythm sleep disorders and neuro-psychiatric illness: A systematic review and meta-analysis. Sleep Med Rev [Internet]. 2019;46:108-23. Available from: https://doi.org/10.1016/j.smrv.2019.04.012

40. Cajochen C. Alerting effects of light. Sleep Med Rev [Internet]. 2007 Dec [cited 2014 Nov 4];11(6):453-64. Available from: http://www.ncbi.nlm.nih.gov/pubmed/17936041

41. Xu Q, Lang CP. Revisiting the alerting effect of light: A systematic review. Sleep Med Rev [Internet]. 2018;41:39-49. Available from: https://doi.org/10.1016/j.smrv.2017.12.001

42. Braun V, Clarke V. Using thematic analysis in psychology. Qual Res Psychol. 2008;3(2):77-101.

43. Terry CG, Hayfield N, Clarke V, Braun V, Terry G, Hayfield N, et al. The SAGE handbook of qualitative research in psychology thematic analysis Introduction. 2020.17-36 p.

44. Gale NK, Heath G, Cameron E, Rashid S, Redwood S. Using the framework method for the analysis of qualitative data in multidisciplinary health research. BMC Med Res Methodol [Internet]. 2013;13(117). Available from:

http://www.pubmedcentral.nih.gov/articlerender.fcgi?artid=3848812\&tool=pmcentrez\&rendertype=abstract

45. www.RE-AIM.org [Internet]. 2019 [cited 2019 Jun 17]. Available from: www.re-aim.org

\section{Figures}

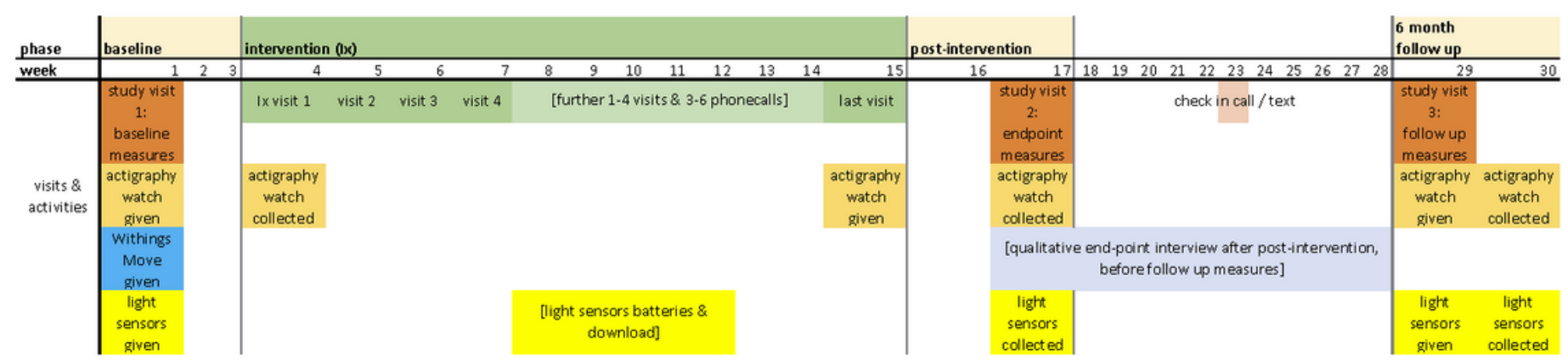

\section{Figure 1}

Research participation timeline 


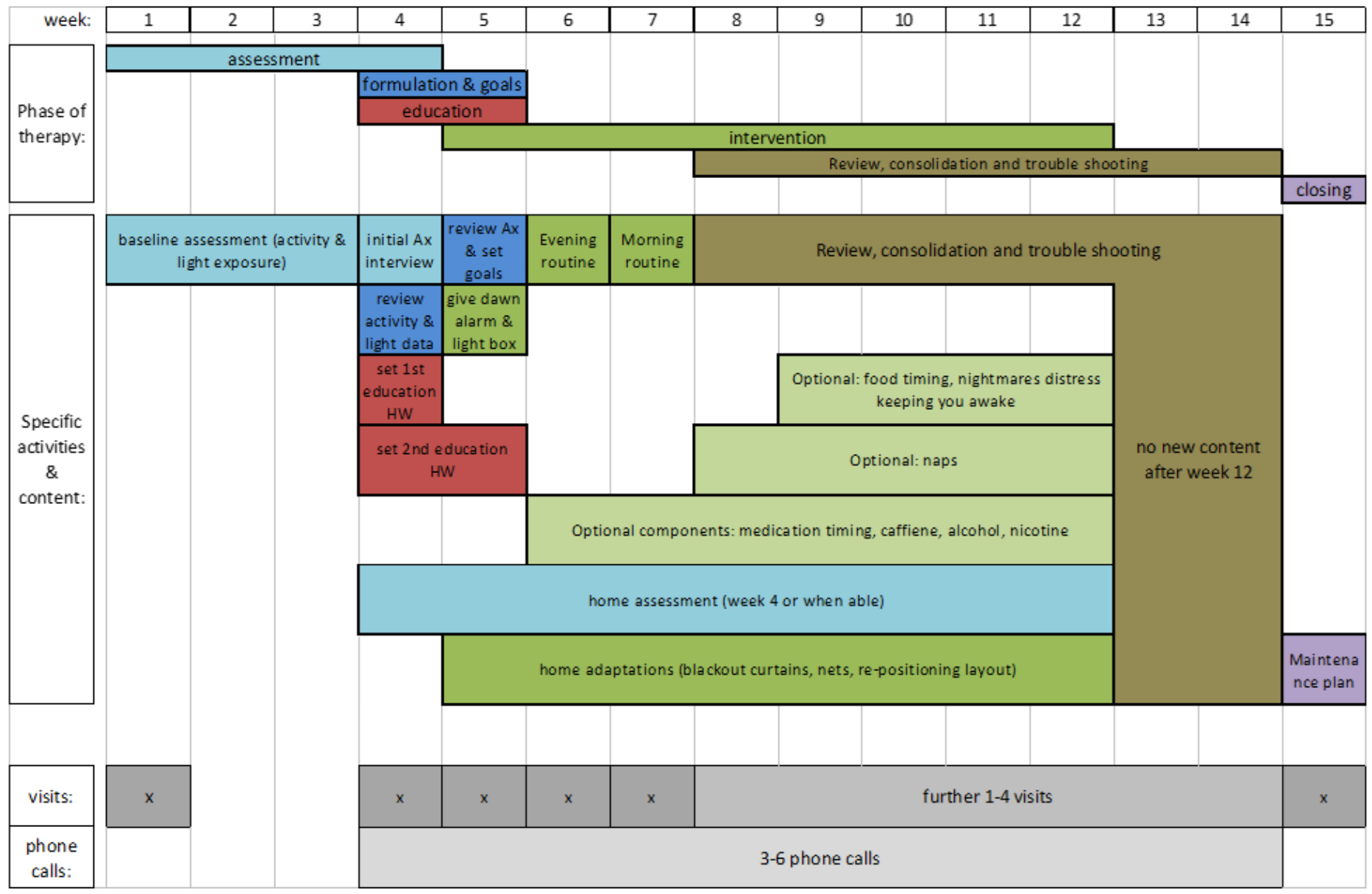

\section{Figure 2}

Therapy content and progression per week of study

\section{Supplementary Files}

This is a list of supplementary files associated with this preprint. Click to download.

- SPIRITcompletedchecklist.doc 